\title{
Research on Scheduling Optimization of Cloud Computing Resource Load Based on Culture Firefly Algorithm
}

\author{
Kexin Zhang ${ }^{1, \text { a }}$ \\ ${ }^{1}$ Jilin Huaqiao University of Foreign Languages Changchun, People's Republic of China \\ a574915363@qq.com
}

Keywords: Firefly Algorithm; Cloud Computing; Resource Load Scheduling Optimization

\begin{abstract}
Cloud computing is a new computing technology with great potential value. It utilizes large-scale hardware and virtual resources to provide users with dynamic application services. In order to maximize the use of cloud resources, give full play to the maximum potential of cloud computing, mining efficient resource scheduling strategy is our top priority. By comparing with the classic function, the optimized algorithm has a great improvement on the search precision and convergence speed. Firefly algorithm can effectively improve the performance of resource scheduling in cloud computing, shorten the time to complete the task, improve the overall processing capacity of the system. On the basis of this improved idea, the problem of balanced network load and extended network can be solved better, the global convergence of the algorithm is improved, and the effect of network operation is enhanced to find the optimal scheduling scheme.
\end{abstract}

\section{Theoretical Introduction of Cloud Computing}

Cloud computing is expressed as an Internet-based computing that provides shared software, resources, and information to computer devices in an on-demand fashion. Cloud computing is a new business computing model. It can allocate computing tasks to resource pools composed of a large number of computing resources, so that users can obtain computing resources, storage space and other services according to their own needs. In general, the nature of cloud computing is self-maintenance management of virtual resources into the virtual pool, including storage service resources, computing resources, bandwidth resources, usually embodied in large-scale server clusters. The resource pool can manage and allocate the resources through the special middleware, thus offer the special-purpose calculation service for the user.

Users can dynamically apply for resources according to their own needs to support the operation of the application, and we do not need to spend too much energy consumption allocation management, which can focus more on their own business, improve efficiency while reducing the cost of work. From a broad perspective, cloud computing can be understood as a service delivery model, that is, through the network to obtain the required services, the service has a large scale, high reliability and so on. Therefore, the cloud computing can be summarized as the unified management of computing resources and scheduling, and on-demand to provide resources to users of the service model ${ }^{[1]}$.

Cloud computing architecture can be divided into three levels: the cloud computing infrastructure, cloud computing application development and execution environment and cloud computing applications. The cloud computing infrastructure includes both infrastructure and infrastructure management. Infrastructure refers to the data center hardware and software, which uses the network as the transmission carrier of information to achieve distributed deployment. Common infrastructure is server, data center network, power and other hardware devices as well as the server operating system as the representative of the software. The core of the cloud computing infrastructure is the management of the infrastructure, which implements the management of the underlying hardware and software co-ordination. Cloud computing applications provide good computing power, storage and other service support. 


\section{Theoretical Analysis of Firefly Algorithm}

Load balancing is an important issue in cloud computing research. Because of the problems of low efficiency, low accuracy and dynamic change of resource demands, a load balancing model is established in cloud computing environment. By introducing chaos algorithm into sender strategy and introducing receiver strategy into receivers' Firefly algorithm to improve the optimization of the target node selection and transfer of the accuracy of the task. The firefly algorithm can prevent the convergence too quickly and the objective function value can easily fall into the local optimum by improving the way of obtaining the fluorescence value. By the simulation test of the three functions, the search precision and performance are significant ${ }^{[2]}$. Fig.1 shows the advantages of firefly algorithm.

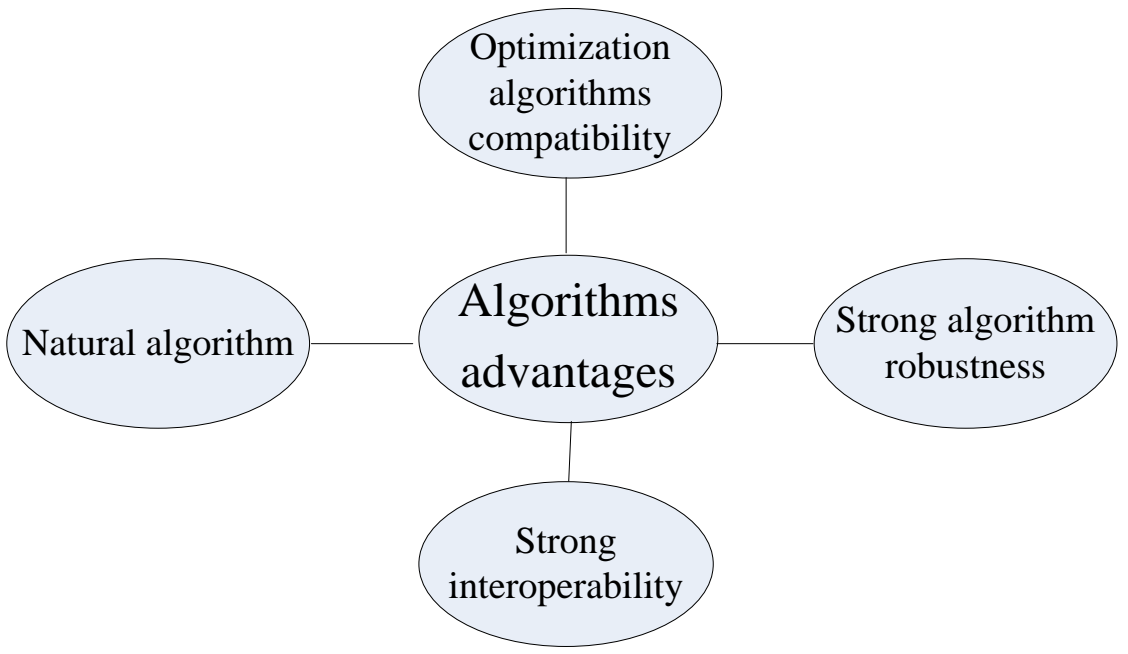

Fig.1.The advantages of firefly algorithm

From the firefly algorithm description and analysis can be seen, firefly algorithm has the followed characteristics: it is a natural algorithm. Although the behavior of single individual is simple, the collective action can show the remarkable effect, and the algorithm produces the best result. Firefly individuals rely on fluorescence has a strong ability to work together. Firefly individuals tend to move to the optimal location, the brightest individual, and the optimal individuals move randomly to find more locations, so that the entire population can have a positive feedback mechanism to ensure that the whole population can find the optimal solution with larger probability. The algorithm is robust. General individual to the best individual movement, the best individual random search, so that the firefly population optimization without prior knowledge, with strong robustness and adaptability. The reason why the firefly algorithm can find the optimal solution quickly is that the feedback mechanism is formed by the information exchange between the individuals in the firefly, which accelerates the convergence of the algorithm and improves the probability of the best solution. If the final condition is satisfied, the optimization process ends, otherwise the steering step continues to be optimized. According to the optimal individual, we get the optimal cloud computing scheduling scheme ${ }^{[3]}$.

Resource scheduling in the cloud computing environment can be divided into the following steps: Resource request phase: Resource request is one of the independent modules of resource scheduling. There are certain resource limits when making a resource request. Resource Discovery Phase: At this stage, we examine all resource pools and gather useful information to build a list of available resources. Resource selection phase: select the appropriate resource from the list of available resources. Usually, at this stage, a certain scheduling algorithm is used to evaluate resource detection results, and resources are selected according to certain scheduling functions. Resource monitoring phase: The system provides resources optimized for the client to process task requests. At the same time, the system monitors that part of the resource. When the use of resources anomalies, re-allocation or removal of resources to ensure the normal use of the user. When the task execution is complete, the scheduled resources are reclaimed. 


\section{Mathematical Model of Firefly Algorithm in Cloud Computing}

Common cloud resource scheduling optimization objectives are the following: according to their business needs to apply for resources, resource usage in accordance with market principles to charge the appropriate fees. The optimal span is the amount of time it takes to complete a user's task in a cloud computing system. From the user perspective, as short as possible to receive the task of cloud system feedback results, good scheduling strategy, the shorter its span. Quality of Service: Any service to provide high-quality measurement standards are quality of service. Cloud computing in the provision of cloud services to customers need to ensure maximum quality of service, in order to reflect the performance of cloud systems. Load balancing is one of the measurement factors of parallel computing and distributed computing resource scheduling algorithm. Cloud data center resources are heterogeneous, to ensure that the load balancing of computing nodes is the core issue of cloud resource scheduling.

Using the randomness, ergodicity and regularity of the variables to optimize the search, the algorithm can jump out of the local optimal, maintain the diversity of groups, and to improve the global search capabilities. In order to prevent this phenomenon from happening, the individuals in the firefly are in the worst position of the chaotic perturbation, which leads to the loss of the difference between the individual fireflies, which is getting closer and closer with the number of iterations. Different chaotic maps have great influence on the process of chaos optimization, and the fireflies chaotic map Logistic is used more frequently. But it has higher probabilities in two ranges, and the optimal speed is affected by the nonuniformity of the traversal. reduce ${ }^{[4]}$. Fig.2 shows optimal result graph.

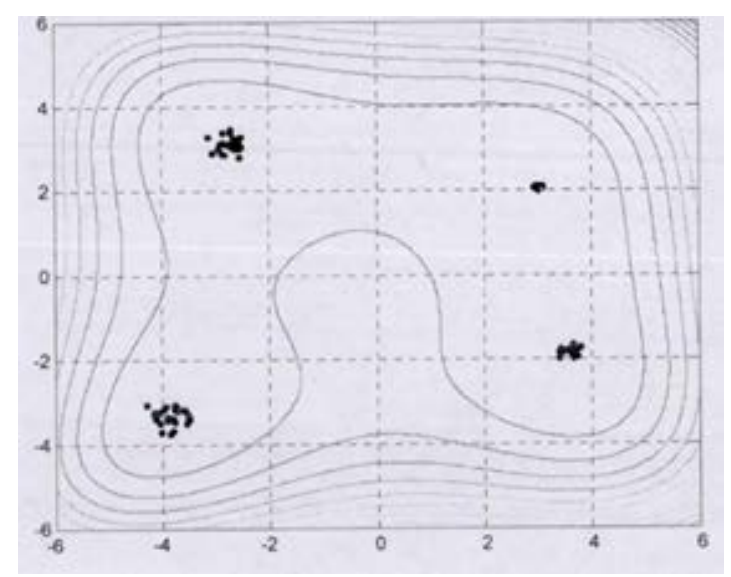

Fig.2. Optimal result graph

In contrast, the cloud computing resources in the scheduling needs of the rate of more factors, first of all on-demand model to provide the appropriate service, then the different business needs to deploy different resources. Second, due to the external nature of cloud computing, cost is also one of the factors considered. At this stage of the cloud environment, resource scheduling technology is not yet mature, but cloud service providers have the problem as the focus of the study. The advantages and disadvantages of resource scheduling strategies and algorithms are the direct factors of system performance.

\section{Application of Firefly Algorithm in Load Resource Optimization}

Resource optimal scheduling refers to the heterogeneous resources in the cloud data center allocated to a number of cloud applications do not affect each other, making the business to complete a short time, the dual objectives of high resource utilization. Cloud resource allocation issues related to the specific requirements of cloud customers, usually with the following constraints: the order of each cloud client given. Each time period can only receive a request for a client task, each client task can only be occupied by a server in the cloud server, once the start can't be interrupted. In the entire process, each task in the same cloud server can only be executed once ${ }^{[5]}$.

In the basic firefly algorithm, the value of fluorescein is to determine whether the location of 
the firefly is the best location, when in the best position, you can guide more fireflies to their own close to find the function of the mouth . Using the improved value of the updated fluorescein can better avoid falling into the local convergence, which can better find the best location, this improvement is more in line with the multi-tasking resources in the rational use of cloud computing. There is no doubt that the completion time between the tasks in the cloud server will be reasonably close to the processing time, thus ensuring the objective function to a minimum. The improved firefly algorithm is applied to the cloud computing. All processing tasks are carried out using the string encoding, randomly select the customer tasks to be processed in the cloud, all tasks are numbered, each firefly represents a solution, the location of fireflies The length indicates all the operations, and the number of fireflies represents the number and space of the solution. Firefly's goal function is the value of the cloud computing task completion time minimum value.

Initialize the parameters of the algorithm, and define the location of the initial fireflies, the definition of the minimum and maximum fitness. For each firefly, the value of the new fluorescein is calculated and the target value of the firefly is the value of the fluorescein. The value of the new fluorescein is judged and controlled by a calculation strategy. For fireflies to transform the location, select the eligible fireflies. Randomly select the direction of the fireflies to update. The next iteration process is performed to determine whether the iteration number satisfies the end condition. If the step is not satisfied; if satisfied, the optimal solution is directly output.

\section{Conclusions}

Cloud computing is the forefront of scientific and technological development in recent years, it is a collection of distributed computing, virtualization technology, grid computing and Web, services and other technologies developed to ensure that cloud users at any time, any place to maximize the use of Cloud computing resources, which can handle large-scale computing problems. In this paper, the resource scheduling of the artificial firefly algorithm is mainly carried out by the virtual machine to implement the dynamic and automatic allocation of resources. The weighted round robin method and the least load first algorithm are used to load balance the physical server resources. Artificial firefly algorithm can improve the location of the strategy of fireflies, so that the fireflies can quickly find a good mouth mark. It is found that the algorithm can effectively reduce the average completion time of the sub-tasks and improve the efficiency of task processing, and achieve the reasonable allocation of resources under the cloud computing model.

\section{Acknowledgements}

This article is a stage result of two larger programme under the Construction and Application of Micro-classes on Office Automation Practice Teaching and the Study of the Sustainable Development of Students' Associations in Private Colleges and Universities

\section{References}

[1] A. H. Gandomi, X. S. Yang, A. H. Alavi. Mixed variable structural optimization using Firefly Algorithm[J]. Computers \& Structures, 2011, 89(23-24): 2325-2336.

[2] G. K. Suyanto. Evolutionary Discrete Firefly Algorithm for Travelling Salesman Problem [J]. Adaptive and Intelligent Systems, 2011, 6943: 393-403.

[3] M. Bidar, H. R. Kanan. Modified Firefly Algorithm Using Fuzzy Tuned Parameters [J]. 13th Iranian Conference on Fuzzy Systems (Ifsc), 2013.

[4] K. Chandrasekaran, S. P. Simony N. P. Padhy. Binary real coded firefly algorithm for solving unit commitment problem [7]. Information Sciences, 2013, 2A9: 67-84.

[5] S. Talatahari, A. H. Gandomi, G. J. Yun. Optimum design of tower structure using Firefly Algorithm [J]. Structural Design of Tall and Special Buildings, 2014, 23(5): 350-361. 